\title{
POWERING BODY AREA NETWORKS USING THE BODY AS A TRANSMISSION MEDIUM
}

\author{
P. Wang ${ }^{l}$, S. X. Li ${ }^{l}$, C. A. Zorman ${ }^{l}$, P. X.-L. Feng ${ }^{l}$, and W. H. Ko ${ }^{1 *}$ \\ ${ }^{1}$ Department of Electrical Engineering \& Computer Science, Case School of Engineering, \\ Case Western Reserve University, Cleveland, $\mathrm{OH}$ 44106, USA
}

\begin{abstract}
Body area networks (BAN) are an enabling technology for individually monitoring health or delivering care in a real-time, effective, and minimally obtrusive way. An ideal BAN requires both a high efficiency signal and power bus. Recent research shows promising signal transmission efficiency using the body as a transmission medium. However, these BAN systems require the sensor nodes to carry on-board power or use through-air transmitted power, whose utility is limited by the power transmission efficiency and/or distance. This paper reports an initial study to investigate, design and evaluate a through-body power transmission system that can distribute power to sensor nodes in the network. Our preliminary results show that it is feasible to distribute $0.5-1.0 \mathrm{~mW}$, at $50 \mathrm{MHz}$, to sensor nodes over a distance of $40 \mathrm{~cm}$ with $\sim 18 \mathrm{~dB}$ loss, which is $\sim 10-100$ times more efficient than through-air transmission. This result is instrumental for realizing a through-body signal and power bus for next generation BAN.
\end{abstract}

\section{INTRODUCTION}

The successful integration of MEMS with low power integrated circuits and wireless communication technologies has enabled rapid development of body area networks (BAN) for human health applications using the body as a transmission medium for signal bus [1]. As shown in Fig. 1, the ideal BAN needs an efficient power bus and signal bus for a central master station to interact with an array of sensor nodes wirelessly. However, these current systems require the sensors to carry onboard power or use through-air transmitted power [2]. On-board power has a lifetime limit while through-air transmitted power is limited by the transmission efficiency and the need for a nearby transmission station.

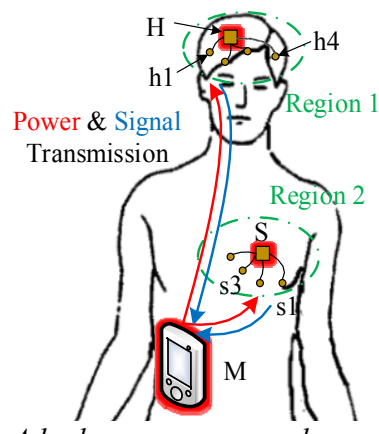

Figure 1: A body area sensor and power network.

It was predicted in 1997 [3] that a power and signal bus might be realized in a through-body medium. Recent reports [4] using computer simulation show the feasibility of powering the sensors wirelessly using the body as a transmission medium. However, these simulations have not been realized experimentally due to several challenges, such as electrode design, power transmission router selection, impedance matching, and low power sensor node electronics design.
This paper reports an initial study to investigate, design and evaluate a power transmission system for clinical applications that can distribute power to operate all sensor nodes in the network. First, we characterized and selected multiple potential routes for BAN signal and power transmission, such as from foot to head, abdomen to chest/head, wrist to chest/upper-arm etc. Second, we improved the design of a BAN electrode widely used for BAN communication [5], and characterized the impedance of this electrode. Third, we designed an impedance matching system based on the electrode properties. Finally, we demonstrated the feasibility of distributing $0.5-1.0 \mathrm{~mW}$, at $50 \mathrm{MHz}$, to sensor nodes over a distance of $40 \mathrm{~cm}$ with $18-23 \mathrm{~dB}$ loss on the human body surface, which is $\sim 10-100$ times more efficient than through-air transmission. In addition, we also report our early stage implant-toimplant power transmission characterization in a vivo animal experiment, which is, to the best of our knowledge, the first published experimental data of in vivo implant power transmission that compares galvanic, capacitive, and coil coupling methods.

\section{POWER DELIVERY PATH CHARACTERIZATION}

Multiple potential routes were characterized for human and animal BAN signal and power transmission. These routes are needed for clinical and medical research purposes.

\section{Measurement Setup}

Transmission loss in a body area sensor network was studied using the configurations shown in Fig. 2. A network analyzer (4395A Agilent Inc.) was used for transmission loss measurements and balun (FTB-1-1 A15+, Mini-Circuits Inc.) was used for isolation. Both galvanic and capacitive coupling methods were evaluated. The transmitter (TX) and receiver (RX) terminals consisted of two electrodes (signal and ground). For galvanic coupling, the two electrodes were attached directly to the skin surface. For capacitive coupling, all signal electrodes were connected to skin and all ground electrodes were floating in air. The transmission routes and directions are shown in Fig. 2-c. The TX and RX electrodes were connected to the network analyzer. During this stage, we adapted the network analyzer to compensate for the mismatch between the electrode and body tissue, due to the
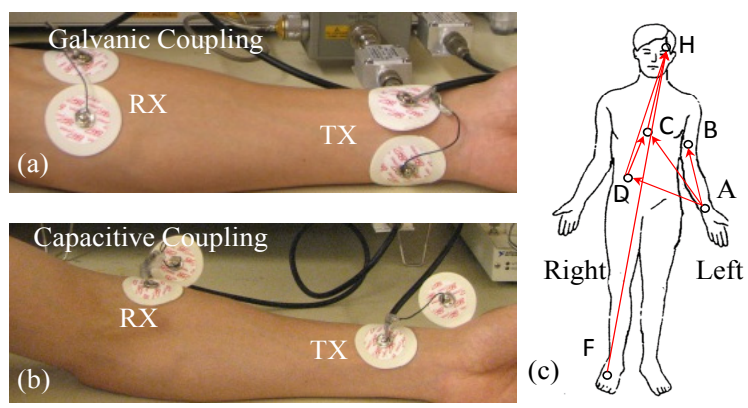

Figure 2: Photos of (a) galvanic and (b) capacitive coupling setups. (c) Transmission directions for $S_{21}$ measurements are marked FH, DH, $C H, A D, A C$, and $A B$. 
difficulty of matching impedance over a wide frequency range.

\section{On-Body Power Transmission Loss}

With the test method shown in Fig. 2, we measured the human body area $S_{21}$ versus frequency $(10 \mathrm{~Hz}-500 \mathrm{MHz})$. The $S_{21}$ characteristics are shown in Fig. 3-a, b and corresponding to the routing shown in Fig 2-c. The line marked "air" is the reference $S_{21}$ with all electrodes in air at a distance of $15 \mathrm{~cm}$. For both galvanic and capacitive coupling, the $S_{21}$ is insensitive to distance for frequencies between 40 and $60 \mathrm{MHz}$. The lowest $S_{21}$ values are $6 \mathrm{~dB}(\sim 380 \mathrm{MHz})$ and $-11 \mathrm{~dB}$ for galvanic and capacitive coupling, respectively. Galvanic coupling at $\sim 380 \mathrm{MHz}$ has a $-6 \mathrm{~dB}$ loss but varies more with distance. For capacitive coupling, the $S_{21}$ has a peak at $\sim 50 \mathrm{MHz}$ of $\sim-11 \mathrm{~dB}$, and differs from through-air transmission by as much as $70 \mathrm{~dB}$. Moreover, the $S_{21}$ has a low sensitivity to a change in TX and RX distance from $15 \mathrm{~cm}$ to $140 \mathrm{~cm}$, suggesting that capacitive coupling might be a feasible means of power distribution for a whole body BAN.
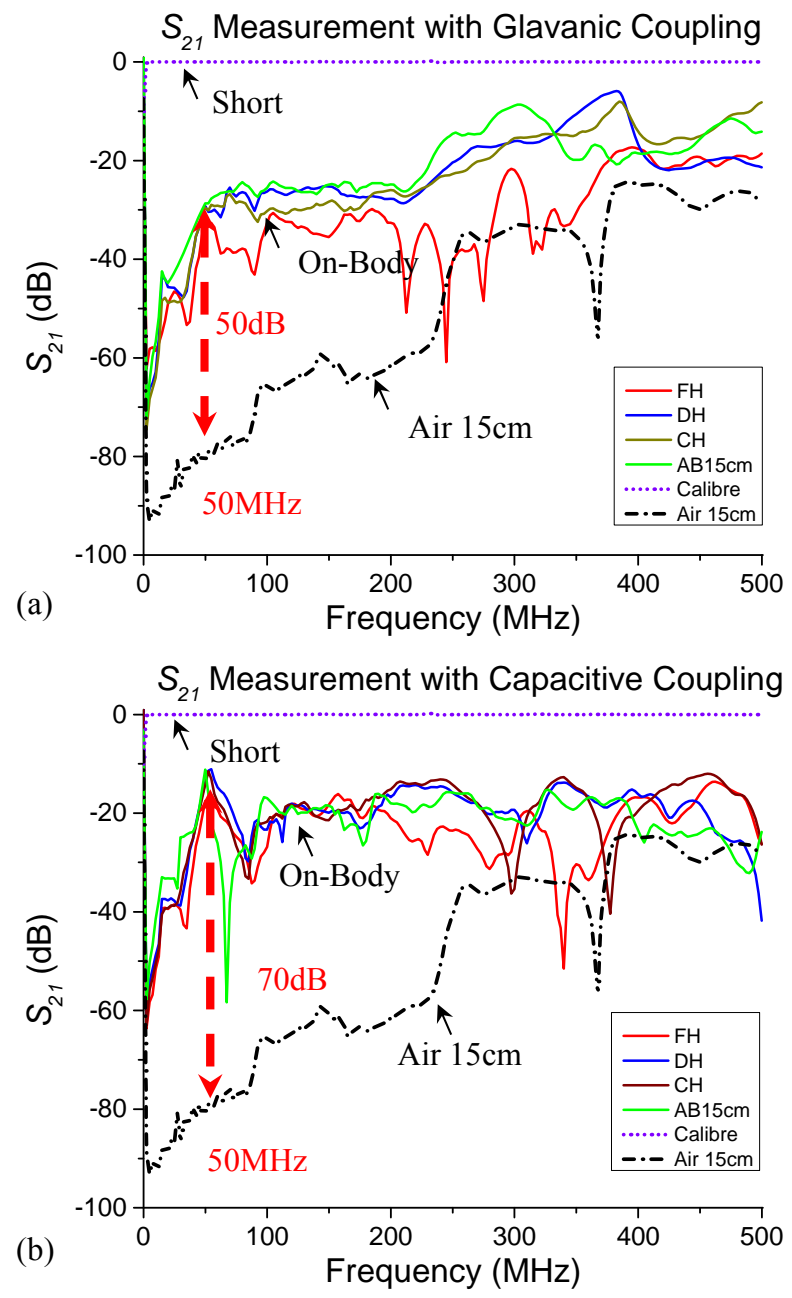

Figure 3: On-body $S_{21}$ measurements for (a) galvanic and (b) capacitive coupling. The positions measured are shown in Fig. 2 (c). "Short" is a baseline with TX/RX shorted.

\section{Implant Power Transmission Loss}

In 2006, we demonstrated the feasibility of through-body signal and power transmission between implanted components in rabbit experiments [6]. In this study, a 5 month-old $8 \mathrm{lb}$ rabbit was used for the experiment. The measurement was conducted with a function generator and oscilloscope. The selected routes are shown in Fig. 4-AB, CD, using a distance of $15 \mathrm{~cm}$.

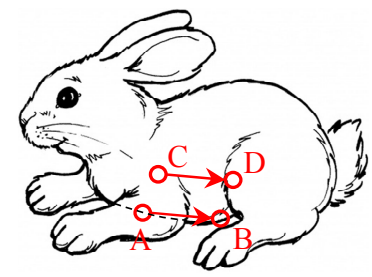

Figure 4: Route of rabbit experiment. AB: on surface skin from near Heart to stomach, and CD: implanted from near Heart to near stomach. Both $A B$ and $C D$ have a $15 \mathrm{~cm}$ distance.

The electrodes used are described in Table 1. Two types of electrodes were used including a 6 turn, a $0.9 \mathrm{~cm}^{2}$ planar coil and an $\mathrm{Ag} / \mathrm{AgCl}$ plate. For the $\mathrm{Ag} / \mathrm{AgCl}$ electrode, $0.6 \mathrm{~cm}^{2}$ and $0.9 \mathrm{~cm}^{2}$ plates were used.

Table 1: Summary of electrodes used in the implant power transmission experiment.

\begin{tabular}{|c|c|c|}
\hline Electrode & Type & Dimension \\
\hline \hline $\mathrm{L} 2$ & Planar coil & 6 turns, $0.9 \mathrm{~cm}^{2}$ \\
\hline $\mathrm{C} 1$ & Planar $\mathrm{Ag} / \mathrm{AgCl}$ plate & $0.9 \mathrm{~cm}^{2}$ \\
\hline $\mathrm{C} 2$ & Planar $\mathrm{Ag} / \mathrm{AgCl}$ plate & $0.6 \mathrm{~cm}^{2}$ \\
\hline
\end{tabular}

The measured $S_{21}$ parameter is shown in Fig. 5. As before, we focus the analysis on the higher frequency range of the data set. The $S_{21}$ at frequencies higher than $50 \mathrm{Mhz}$ might show a similar result when using balun for isolation, which is at a frequency range of $50-100 \mathrm{MHz}$ in this case. Within this frequency range, Fig. 5shows that all $S_{21}$ curves have a peak near $86 \mathrm{MHz}$. The $\mathrm{C} 1$ $\mathrm{Ag} / \mathrm{AgCl}$ electrode has losses of $23 \mathrm{~dB}$ and $31 \mathrm{~dB}$ for on-skin and implant respectively. The larger $\mathrm{Ag} / \mathrm{AgCl}(\mathrm{C} 1$ than $\mathrm{C} 2)$ electrode shows a 7 and $11 \mathrm{~dB}$ less loss for on-body and implant respectively. The $\mathrm{Ag} / \mathrm{AgCl}$ electrode ( $\mathrm{C} 1$ implant) shows a $16 \mathrm{~dB}$ less loss than the planar coil (L2 implant) with the same area at $86 \mathrm{MHz}$.Although the grounding, impedance matching and isolation issues were not addressed due to the limited instrumental resources at that time, the reported $S_{2 I}$ data still offer worthwhile information. For the balun isolation affect for frequencies below $50 \mathrm{MHz}$ according to Xu's experiment [7], and the transmission

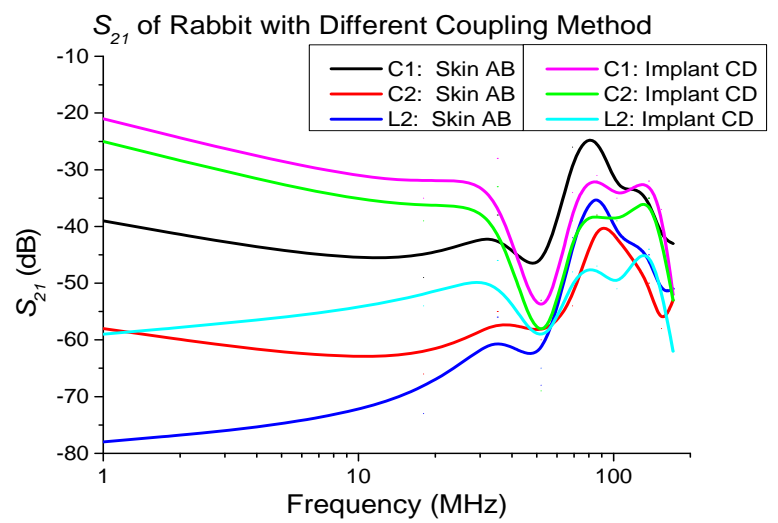

Figure 5: Comparison of measured $S_{21}$ versus frequency for implanted and surface mounted electrodes in a rabbit model spaced $15 \mathrm{~cm}$ apart from heart to stomach. Cl is a $0.9 \mathrm{~cm}^{2}$ electrode; $C 2$ is a $0.6 \mathrm{~cm}^{2}$ electrode; L1 is a $0.5 \mathrm{~cm}^{2}, 4$ turn coupling coil; L2 is a $0.9 \mathrm{~cm}^{2}, 6$ turn coupling coil. 
loss at higher frequencies will be less if impedance was matched.

\section{ON-BODY POWER TRANSMISSION}

\section{Electrode Types}

Fig. 6 shows the diagram and dimension of the electrode designed for on-body power transmission. The design described in Ref.5 was adapted and improved by adding a matching network between the electrode and feed line and using a wide copper ribbon for connection. The matching network reduced the reflection coefficient $\Gamma$, and the wide ribbon reduced the high frequency impedance which is beneficial for power transmission.

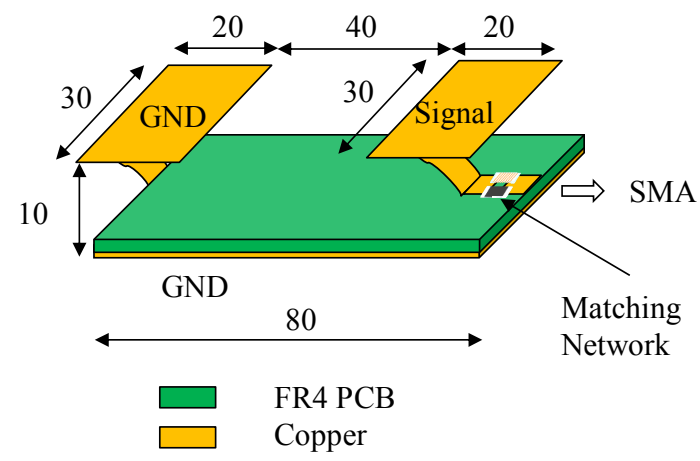

Figure 6: Diagram of the electrode for on body power transmission experiment. Fixed LC matching network was applied near the feed line connection. All dimensions are in $\mathrm{mm}$.

\section{Impedance Matching}

In order to realize good impedance matching, we modified the electrode design of [5], by adding a matching network between the electrode and feed line and replacing the pillar with a wide ribbon copper (Fig. 6). Impedance matching is needed: i) to reduce the reflection between body tissue and wearable electronics; ii) to convert the received weak AC energy into a feasible DC supply.

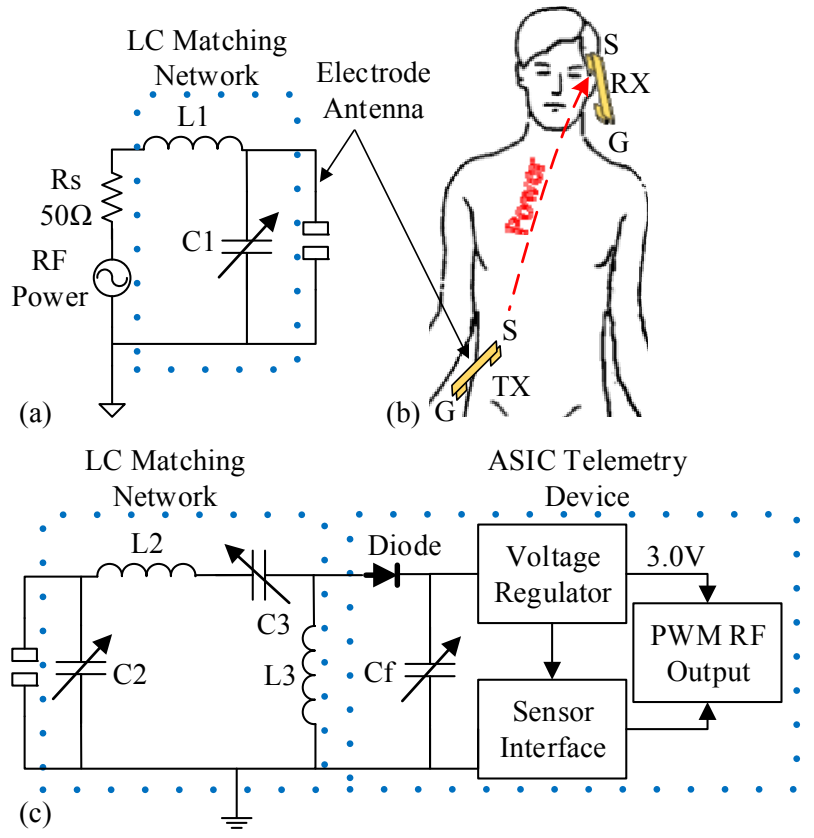

Figure 8: Circuit diagram of preliminary power distribution experiment; (a) TX; (b) Experiment setup for (a) and (c). TX and $R X$ electrode pairs are configured to the abdomen and head by capacitive coupling; (c) Sensor node device.
We implemented the matching network in three steps: 1) matching the master station (chest) electrode to the $50 \Omega$ output RF function generator (AFG3252, Tektronix Inc.); 2) matching the receiver (i.e., head) electrode to a $50 \Omega$ transmission line; 3) matching the $50 \Omega$ transmission line to the low dropout rectifier with the load cascaded. Steps 2 and 3 can be merged to one step to reduce the mismatch due to the calculation error and stray electrical parameter. We separate step 2 and 3 for convenient RF measurement. The matching network in Fig. 8 was realized by the fixed passive LC component. The LC-type matching network was chosen for its relatively low $\mathrm{Q}$, which is robust for two reasons: i) the proposed powering system adopts a narrow band frequency at $50 \mathrm{MHz}$; ii) the human body impedance varies significantly, which increases the reflected power. The component values for matching a fixed AC load and DC load after the rectifier was calculated according to conjugate-image impedance theory [8] and the equivalent rectifier model $[9,10]$ respectively. The estimated reflected power is less than $1 \%$, according to $<0.1$ reflection coefficients $\Gamma$ (Fig. 9)

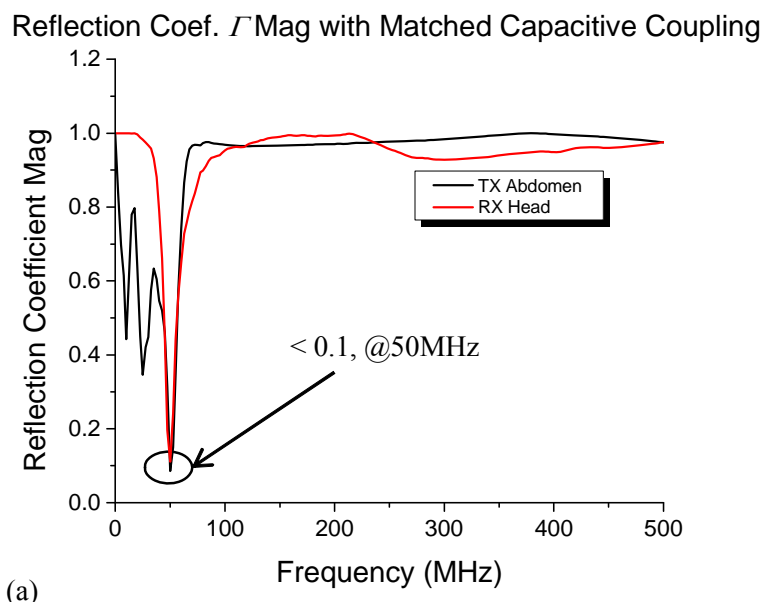

(a)

Reflection Coef. $\Gamma$ Phase with Matched Capacitive Coupling

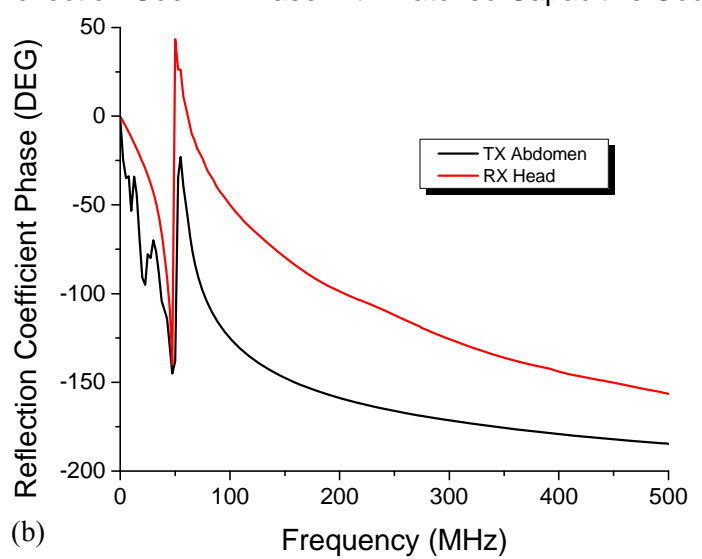

Figure 9: Impedance matching result, measured on the SMA feed point (Fig. 6) when electrode was coupled to human body capacitive. (a) and (b) are magnitude and phase of the reflection coefficient for TX/RX electrode placed on abdomen and head. All data comes from measurement after impedance matching.

\section{Powering Actual Sensor Node Device}

Using the configuration in Fig. 8, we investigated the possibility of a capacitive coupling-based distribution network to supply operating power from a master station to sensor nodes. 
Measurements were made both with a fixed $10 \mathrm{k} \Omega$ resistor load and an implantable blood pressure telemetry ASIC device (Fig. 8-c). The $10 \mathrm{k} \Omega$ is an equivalent load to the ASIC device previously developed by our group that uses $200 \mu \mathrm{A}$ to charge a thin film battery [11]. The $50 \mathrm{MHz}, 50 \Omega$ output RF power source was at 18 $23 \mathrm{dBm}$. The coupling electrode is shown in Fig. 6. The distance from TX to RX electrodes was $\sim 40 \mathrm{~cm}$. Balun was used to isolate the body from the instrument. LC networks were used for matching the $50 \Omega$ RF source, the body impedances $(\sim 100-1113 \Omega)$, the $10 \mathrm{k} \Omega$ load and ASIC device rectifier input. The received voltage at $10 \mathrm{k} \Omega$ load and output of ASIC device regulator can reach $3 \mathrm{~V}$, and the received power was $\sim 0 \mathrm{dBm}(1 \mathrm{~mW})$, which is capable of charging a $50 \mu \mathrm{Ah}$ lithium battery with $100 \mu \mathrm{A}$ current or to simultaneously power tens of $3 \mu \mathrm{W}$ pressure telemetry units. The estimated total transmission loss was $\sim 18-23 \mathrm{~dB}$ for the $10 \mathrm{k} \Omega$ load and ASIC device respectively. The greater loss of the ASIC device as compared with the $10 \mathrm{k} \Omega$ resistance load might be due to the loss on the diode stage, which can be improved by adding a voltage multiplier stage before the rectifier.

\section{CONCLUSION}

This paper reports an initial study to investigate, design and evaluate a power transmission system that can distribute power to operate all sensor nodes in the network. Several challenges are addressed for realizing power transmission using the body as a medium, such as electrode design, power transmission route selection, impedance matching, and low power sensor node electronics design. An example design was introduced to address these challenges. The preliminary result shows that it is feasible to distribute $0.5-1.0 \mathrm{~mW}$ to sensor nodes over a distance of $40 \mathrm{~cm}$ with an $18-23 \mathrm{~dB}$ loss, which is $\sim 10-100$ times more efficient than through-air transmission. The preliminary implant experiment result also shows feasible power transmission with a loss of $\sim 23 \mathrm{~dB}$. These results are helpful for realizing both a though-body medium signal and power bus for next generation BAN.

In the future, the utility and safety of the proposed powering system require the following key advancements. i) The system must be miniaturized for minimally intrusive health monitoring, with a tradeoff between maintaining high transmission efficiency and reducing the coupling ground area. ii) Ultra-low power sensor node devices are required reduce the power budget at power source within safety standards. For example, an $80 \mu \mathrm{W}$ sensor node requires only $-20 \mathrm{dBm}$ power at the master station with a typical $20 \mathrm{~dB}$ path loss; iii) Noise related issues, such as signal/noise ratio (SNR) and interference between powering path and signal transmission path must be addressed. Although not detailed here, we found the RF signal for the proposed powering system is pure in the frequency domain.

We are applying the proposed on-body power transmission method to power a $3-80 \mu \mathrm{W}$ ASIC RF transmitter (Fig. 8-c) that is integrated with a blood/ECG sensor using the through-body medium. The output pulse width modulation (PWM) carrier frequency will be tuned to $380 \mathrm{MHz}$, which is the optimum frequency according to our power delivery path characterization. The signal transmission though-body medium is ongoing. The miniaturization of electrode and adaptive impedance matching will also be addressed in a future study. These works will be reported in future publication.

\section{ACKNOWLEDGEMENTS}

The authors would like to thank S.J.A. Majerus, Z.H. Wang for instrument support and discussing. Thank H.X. Zhang, Y.N. Cheng for animal experiment.

The financial and instrumental support of the study comes from NIH R-21-EB014442 and Louis Strokes Cleveland Medical Center of the Department of Veterans Affairs, the Advanced Platform Technology (APT) Center, and Case School of Engineering.

\section{REFERENCES}

[1] M. Seyedi, B. Kibret, D. T. H. Lai, and M. Faulkner, "A Survey on Intrabody Communications for Body Area Network Applications", IEEE Transactions on Biomedical Engineering 60, 2067-2079 (2013).

[2] J. S. Besnoff, T. Deyle, R. R. Harrison, and M. S. Reynolds, "Battery-Free Multichannel Digital ECG Biotelemetry Using UHF RFID Techniques", IEEE International Conference on $R F I D, 4 / 30-5 / 2 / 13$, Orlando, FL (2013), pp. 16-22.

[3] E. R. Post, M. Reynolds, M. Gray, J. Paradiso, and N. Gershenfeld, "Intrabody Buses for Data and Power", The International Symposium on Wearable Computers, 10/1314/97, Cambridge, MA (1997), pp. 52-55.

[4] K. Ito, N. Haga, M. Takahashi, and K. Saito, "Evaluations of Body-Centric Wireless Communication Channels in a Range from $3 \mathrm{MHz}$ to $3 \mathrm{GHz}$ ", Proceedings of the IEEE 100, 23562363, (2012).

[5] F. Koshiji, K. Sasaki, D. Muramatsu, and K. Koshiji, "Input Impedance Characteristics of Wearable Transmitter Electrodes for Intra-Body Communication", The $1^{\text {st }}$ Global Conf. on Consumer Electronics, 10/2-5/12, Makuhari Messe, Tokyo, Japan (2012), pp. 362-363.

[6] C. A. Zorman, D. J. Young, P. X.-L. Feng, and W. H. Ko, Interal Technical Report (included in NIH R21 proposal), Case Western Reserve University (2011).

[7] R. Y. Xu, H. J. Zhu, and J. Yuan, "Characterization and Analysis of Intra-Body Communication Channel", Antennas and Propagation Society International Symposium, 06/1-5/09, Charleston, SC (2009), pp. 1-4.

[8] S. Roberts, "Conjugate-Image Impedances", Proceedings of the IRE 34, 198-204 (1946).

[9] J. P. Curty, N. Joehl, C. Dehollaini, and M. J. Declercq, "Remotely Powered Addressable UHF RFID Integrated System", IEEE Journal of Solid-State Circuits 40, 2193-2202 (2005).

[10] J. P. Curty, N. Joehl, F. Krummenacher, C. Dehollaini, and M. J. Declercq, "A Model for $\mu$-Power Rectifier Analysis and Design", IEEE Transactions on Circuits and Systems I: Regular Papers 52, 2771-2779 (2005).

[11] P. Wang, D. Sun, S. J. A. Majerus, S. B. Lachhman, S. X. Li, M. S. Damaser, C. A. Zorman, P. X.-L. Feng, W. H. Ko, "Implantable Pressure Telemetry Device With Thin Film Micropackage", Biomedical Engineering Society Annual Meeting, 09/25-28/13, Seattle, WA (2013).

\section{CONTACT}

*W.H. Ko, Tel: +1-216-368-4081; Email: whk@case.edu 